\title{
Sensor Device for Testing Activities in Parkinson and ALS Patients
}

\author{
K. Grandez ${ }^{1}$, G. Solas ${ }^{1}$, P. Bustamante ${ }^{1,2}$, B. Sedano ${ }^{1}$ \\ Electronics and Communications Department \\ CEIT and Tecnun (University of Navarra) \\ San Sebastian, Spain \\ kgrandez@ceit.es, gsolas@ceit.es, pbustamante@tecnun.es, bsedano@ceit.es
}

\begin{abstract}
The diagnosis and treatment of neurodegenerative diseases has become a key point in nowadays European healthcare system. However, the underlying testing and monitorization processes haven't experienced the innovation and advancement required to fulfill the needs that such diagnosis and treatment present. In this paper we propose a new testing device for Parkinson Disease and Amyotrophic Lateral Sclerosis patients. The device is designed to carry out two of the most used tests for these patients: the finger-tapping test and the hand-grip strength test. The designed system has been divided in three main blocks, which have been implemented and tested: a sensorized glove, equipped with Force Sensitive Resistors (FSR), a tiny hardware device which gathers the data and a PC application, which presents the results to the doctors. This work is part funded by the ICT programme of the European Commission (PERFORM Project: FP7-ICT-2007-1-215952).
\end{abstract}

Keywords: Parkinson, ALS, finger tapping, hand grip strength, force sensitive resistor, sensor device, embedded system

\section{INTRODUCTION}

The world's older population has been growing more numerous for centuries, but the pace of growth has accelerated. The global population age 65 or older was estimated at 461 million in 2004, an increase of 10.3 million just since 2003 . Projections suggest that the annual net gain will continue to exceed 10 million over the next decade-more than 850,000 each month. In 1990, 26 nations had older populations of at least 2 million, and by 2000 , older populations in 31 countries had reached the 2 million mark. Projections to 2030 indicate that more than 60 countries will have at least 2 million people age 65 or older[1].

Europe is not an exception. In fact, except for Japan, the world's 20 oldest countries are all in Europe. And it should remain the world's oldest region well into the 21 st century. Moreover, some studies claim that the population ageing will accelerate and that Europe will see its populations continue to age to unprecedented levels over the next 25 years.

The growth in the population age brings the growth of number of diseases. And one of the major group of diseases that affect elderly people is that of neurodegenerative diseases.

The Parkinson Disease (PD) is a disorder of certain nerve cells in the part of the brain which produces dopamine. These nerve cells break down, dopamine levels drop and brain signals

PERVASIVEHEALTH 2010, March 22-25, Munchen, Germany

Copyright (C) 2010 ICST 978-963-9799-89-9

DOI 10.4108/ICST.PERVASIVEHEALTH2010.8867 which are responsible for the movement become abnormal. PD usually begins in the middle or late life (after age 50). It progresses gradually for 10-15 years. This results in more and more disability. Patients suffering from PD present major clinical abnormalities of movement like resting tremor, rigidity, bradykinesia and postural instability.

Amyotrophic lateral sclerosis (ALS), often referred to as "Lou Gehrig's Disease," is a progressive neurodegenerative disease that affects nerve cells in the brain and the spinal cord. Motor neurons reach from the brain to the spinal cord and from the spinal cord to the muscles throughout the body. The progressive degeneration of the motor neurons in ALS eventually leads to their death. When the motor neurons die, the ability of the brain to initiate and control muscle movement is lost. With voluntary muscle action progressively affected, patients in the later stages of the disease may become totally paralyzed.

The cases for Parkinson Disease and ALS are expected to double worldwide by the year 2020 [2]. Proper medical care of these patients is getting increasingly complex and expensive. Lengthy hospital stays for monitoring and adjustment of the patients' treatment and the problems related with it, contribute to cost increase and morbidity because of the hospitalization itself. But there is a clinical deficit of objective data on which neurologists can base the assessment and care of patients with chronic neurologically-based movement disorders.

One of the ways of overcoming this lack of data is developing new easy-to-use testing devices, which can be left in the patient's own home and used to carry out periodic tests without having to go to hospital to do so. The comfortable testing processes and devices make the patients more prone to carry them out, and this leads to a wider amount of data available for the doctors.

Two of the more widely used tests with neurodegenerative disease patients are the Finger Tapping Test (FTT) and the Hand-grip Strength Test. In the case of the FTT [3], the patients is asked to tap two of the fingers of one hand as quick as possible, and the main parameter measured by the doctors is the tapping frequency. On the other hand, in the hand grip strength test [4] the measured parameter is the force the patient is capable of apply when grabbing an object.

In this paper we propose a single tiny portable device which is designed to carry out both of the tests. It is integrated in a 
system consisting of the mentioned device, a sensorized glove which is worn by the patient, and a PC or base station, which is in charge of receiving the data sent by the device, and visualizing them graphically in order to be analyzed by the doctors.

\section{STATE OF THE ART}

The Unified Parkinson's Disease Rating Scale (UPDRS) [5] is a rating tool to follow the longitudinal course of Parkinson Disease. It is made up of the 1) Mentation, Behavior, and Mood, 2) ADL and 3) Motor sections. A total of 199 points are possible. A value of 199 represents the worst (total) disability, and a value of 0 represents no disability. Under the "Motor exam" section, one of the indicators refers to "Finger taps".

The Finger Tapping Test (FTT), originally developed as part of the Halstead Reitan Battery (HRB) of neuropsychological tests, is a simple measure of motor speed and motor control and is used in neuropsychology as a sensitive test for brain damage [6]. Although motor functioning in humans is controlled by many areas of the brain, the motor strip rostral to the central sulcus is most important, and the functioning of this area is reflected directly in the FTT. As well as direct motor effects, the speed, co-ordination, and pacing requirements of finger tapping can be affected by levels of alertness, impaired ability to focus attention, or slowing of responses. Tapping frequency can distinguish patients with motor dysfunctions of cerebella, basal ganglia, and cerebral origins from normal subjects.

At the onset of ALS the symptoms may be so slight that they are frequently overlooked. With regard to the appearance of symptoms and the progression of the illness, the course of the disease may include muscle weakness. Muscle weakness is a hallmark initial sign in ALS, occurring in approximately $60 \%$ of patients. The hands and feet may be affected first, causing difficulty in lifting, walking or using the hands for the activities of daily living such as dressing, washing and buttoning clothes.

ALS is a very difficult disease to diagnose. To date, there is no one test or procedure to ultimately establish the diagnosis of ALS. Methods for the evaluation of strength in people with ALS include a clinical neurological exam, manual muscle testing (MMT)[7], and rating scales. These methods are subjective and lack sensitivity to detect small changes. The purpose of the Hand Grip Stregth Test is to measure the maximum isometric strength of the hand and forearm muscles.

The devices and methods used so far for the proposed tests haven't had any significant improvement or innovation for many years. Traditional ways of performing the tests are still used.

For the finger tapping test, several methods have been proposed and used. The standard method consists of asking the patients to start with the finger tapping process and an examiner using a stop watch to keep track of the 10-second trial interval. Electronic devices which are based on the same testing methodology have been marketed. The electronic device has an internal timer that starts on the first tap and stops counting taps when the 10 seconds have elapsed. The use of automatic timing is intended to increase the accuracy of testing [8].
Other devices used, which can be found in the literature, include precision image-based motion analyzer and passive marker-based movement analyzer [9]; the Halstead-Reitan fingertapping test (HRFTT), developed and manufactured by Reitan Neuropsychological Laboratory [10], which uses an electronic counter and a tapping key; finger tapping devices containing pressure sensors [11]; systems consisting of accelerometers and touch sensor [12] [13].

In the case of the hand-grip strength measurement, the innovations carried out in recent years have been even poorer. The most usual way to carry out this specific test is by using hand-grip and pinch-force dynamometers [14], which offer very poor information about the way the hand grabs objects. Electromyography has been also used in some studies [15].

\section{SYSTEM ARCHITECTURE}

The study of the state of the art shows that the devices and methods developed so far for the testing activities in patients affected by PD and ALS lack of the most important characteristics of the device described in this article. More concretely:

- Accuracy: the data provided by the device show exact values for the parameters the doctors are interested in. They are not based on subjective appreciation of the performance of the tests carried out by the patients.

- Ease of use: both for the clinicians and for the patients. The patient can carry out the tests without having to move from their own homes. And the data is stored in a PC, which offers the possibility of sending it to the hospital via Internet, for the doctors to analyze the results.

- Frequency: the ease of use of the system makes it possible to carry out more frequent tests, so the tracking of the variations of the motor functions of the patients is more accurate.

- Versatility: using the devices presented in this article, several different tests can be performed, and in each of such tests, several parameters can be measured. For example, for the finger tapping case, both the speed and the regularity (periodicity) can be obtained, which enriches the results of the test and enhances the analysis and the conclusions obtained with it.

In order to comply with this characteristics or requirements, in this paper we propose a system which is based on the architecture shown in Figure 1.

The whole system architecture is composed of three main building blocks:

- Sensorized glove: this glove is equipped with five sensors, which are attached to it.

- Hardware device: this is the main development of the present research work. It consists of a tiny electronic circuit, protected by a case specifically designed for it. Its main functions are the acquisition and processing of the signals coming from the sensors, and transmitting them to the PC via the USB connection. 
- $\quad P C$ application: the third component of the system architecture is in charge of receiving the data sent by the hardware device via the USB connection, and storing and visualizing them, using a graphical user interface.

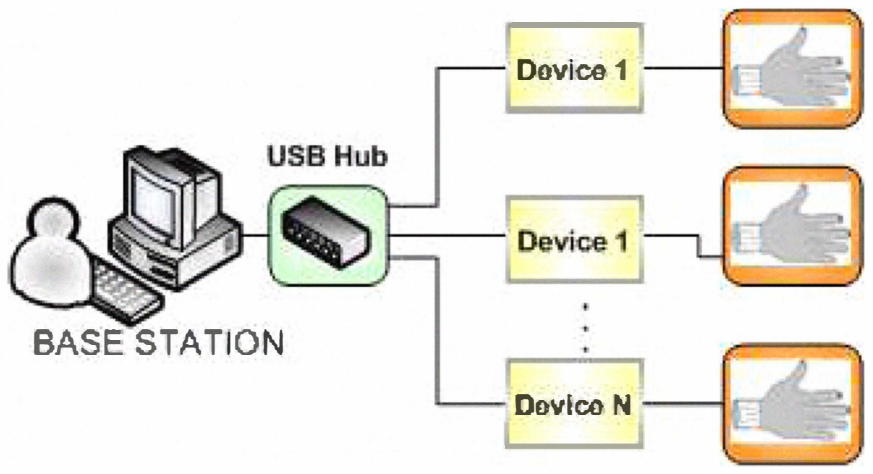

Figure 1. System architecture

The overall way of working of the system is as follows: 1) The patient puts on the glove with the sensors. 2) Connects the glove to the hardware device using the Insulated Displacement Connector (IDC). 3) Connects the hardware device to the PC using a USB cable. 4) Executes the PC application. 5) Starts the signal acquisition using the on/off switch of the hardware device. 6) Carries out the test.

\section{GLOVE AND SENSORS}

As it has been mentioned before, and as can be seen in Figure 2. , the approach followed in this research work has been the one of attaching several sensors to a glove. This design allows complying with one of the key characteristics identified in the system architecture section: the versatility. We consider that this design is more versatile in order to allow carrying out different type of tests and obtaining a wide range of results.

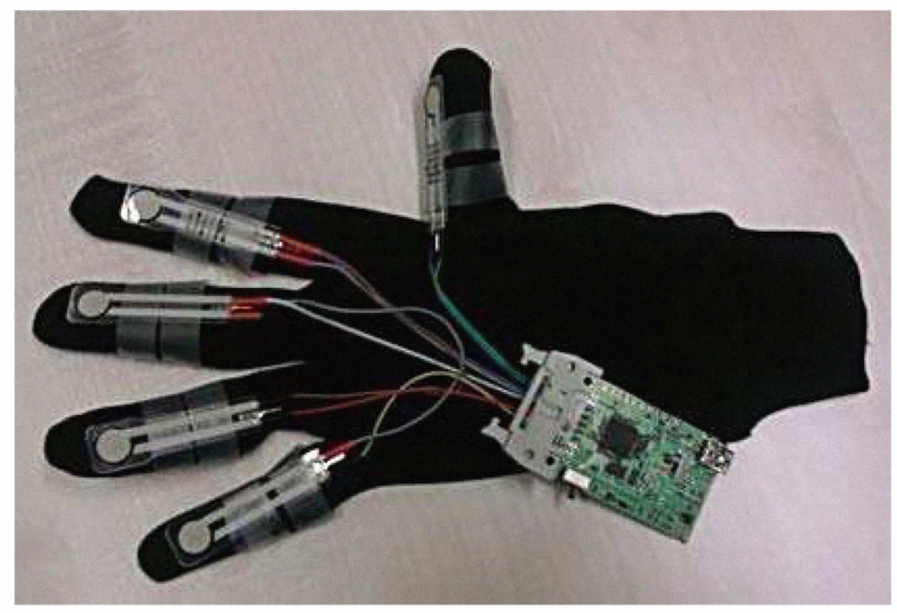

Figure 2. Picture of the sensorized glove

The sensors used in this work have been Force Sensitive Resistors (FSR). A force-sensitive resistor (alternatively called a force-sensing resistor) has a variable resistance as a function of applied pressure. In this sense, the term "force-sensitive" is misleading - a more appropriate one would be "pressuresensitive", since the sensor's output is dependent of the area on the sensor's surface to which force is applied.

The sensors used in this work are manufactured by Tekscan [16], and are constructed of two layers of substrate film. On each layer, a conductive material (silver) is applied, followed by a layer of pressure-sensitive ink. Adhesive is then used to laminate the two layers of substrate together to form the force sensor. The active sensing area is defined by the silver circle on top of the pressure-sensitive ink. Silver extends from the sensing area to the connectors at the other end of the sensor, forming the conductive leads. Figure 3 . shows a picture of the Tekscan FSR sensor.

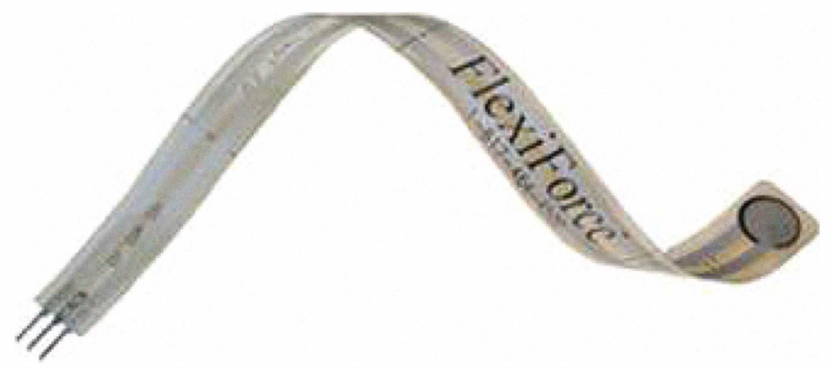

Figure 3. Tekscan FSR sensor.

When external force is applied to the sensor, the resistive element is deformed against the substrate. Air from the spacer opening is pushed through the air vent in the tail, and the conductive material on the substrate comes into contact with parts of the active area. The more of the active area that touches the conductive element, the lower the resistance. When the force sensor is unloaded, its resistance is very high. When a force is applied to the sensor, this resistance decreases.

One of the most common circuits implemented to use an FSR's output is the voltage divider. A voltage is applied to one of the leads, while the other is grounded. FSRs are not polar, meaning it does not matter which side receives the voltage. One lead from a second resistor (with fixed value) is then connected to the voltage side, while the other lead of the second resistor is also connected to ground. In this way the FSR is able to measure the "voltage drop across a resistor". The resistance value of the second resistor determines the output range of the sensor. This is the way in which the signals coming from the sensors are acquired in the hardware device.

The sensors have been mounted by attaching one sensor per finger in the glove. That way, one force signal per finger can be obtained and visualized in the PC application, what makes the doctors to have more information in order to obtain accurate conclusions.

\section{HARDWARE DEVICE}

Data measured from sensors in the glove are processed by the hardware device. This device has to be able to gather this information and prepare it in a data frame in order to relay it to the PC through USB. This whole procedure is represented systematically in the Platform Architecture which gives a better idea of it, see Figure 4. 


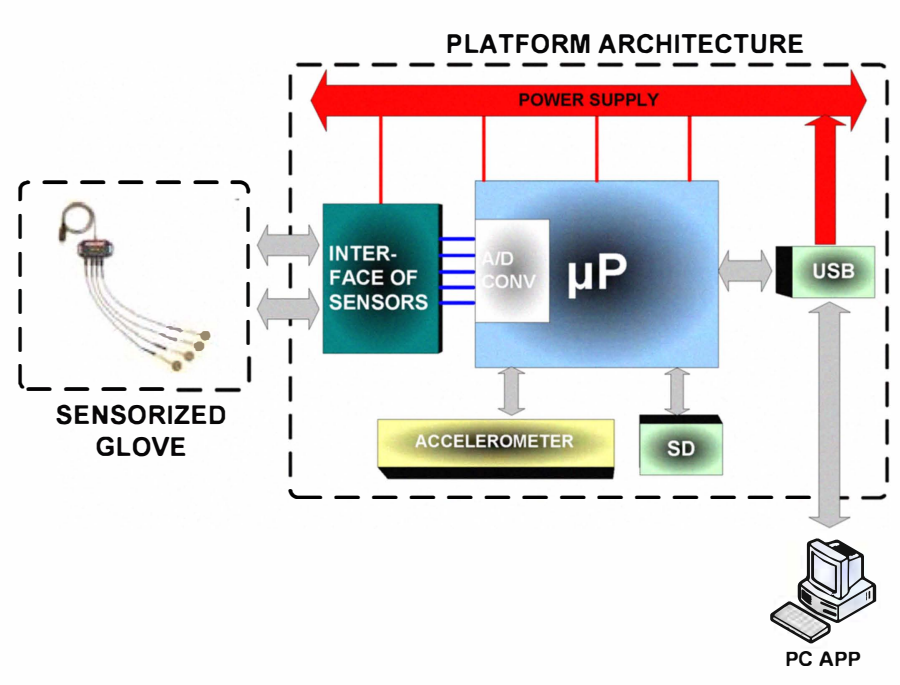

Figure 4. Hardware Architecture

\section{A. Hardware}

The design of the portable device was related to its main functionality explained above. Measurements obtained from sensors in the glove fingers are transmitted through wires to an IDC connector located at one edge of the device. This connector allows connect these inputs to $\mathrm{A} / \mathrm{D}$ channels extended from the CPU. An interface stage is needed for each input due to sensors, done basically with some operational amplifiers and passive filters.

The CPU of the portable device is a Microchip PIC18 Microcontroller with nanoWatt technology. It was chosen due to its maximum number of 13 input $A / D$ channels (it is required to sense 5 signals but node can be extended for further applications), its USB interface (fast and practical transference of data) and for its low power consumption in deep sleep mode, ideal to work as sensor node in monitoring applications.

Analogical input signals attached to the IDC connector are converted into digital up to 10-bit values which are put in an established frame structure according to a particular protocol. The frame arranged is waiting for be transmitted immediately to the PC application for visualization via USB. The transmission of a set of measurements is programmed to be done each $40 \mathrm{~ms}$ but it can be modified using the PC application, from $20 \mathrm{~ms}$ to $50 \mathrm{~ms}$ depending on the resolution and accuracy needed for the clinicians and limited by the A/D conversion latency..

The USB device module is a mini-USB 2.0 compliant allowing fast transmission of data. The platform is supplied by connecting USB so that it is ready to transmit measurements from fingers tapping when USB cable is plugged. Data transmission starts after turning on the switch located at one extreme of the platform, from this instant one of the three LED begins blinking. The device has 3 LEDs which their main functionality is to indicate its states.

The platform is designed to use a 3-axis accelerometer in case it was needed; it also offers the option to record data in a
micro-SD card placed in the bottom side and can be battery powered and recharge through USB. These additional features were included in the design for its using in future work.

\section{B. Embedded Software}

The software developed to be embedded in the platform has a modular scheme. This design allows the software being independent from the platform and also gives flexibility.

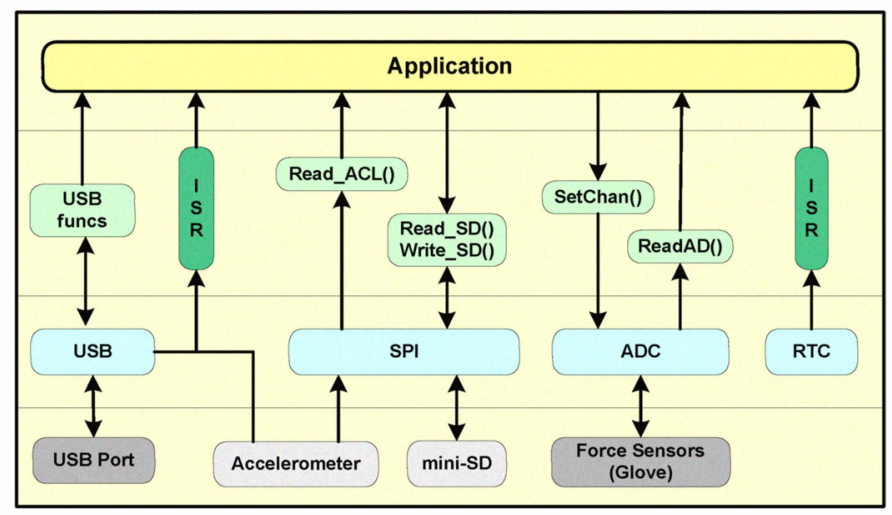

Figure 5. Embedded SW architecture

The whole software structure is divided in 4 layers as it is depicted in Figure 5. The layers are separated by lines and a short description for each one is given below:

- Physical level: is the lowest level and it depends on the hardware directly. The modules present in this level are corresponding to the physical modules of the node; these are the force sensors, accelerometer (not mounted), the USB port and the mini-SD slot (optional).

- Controller level: this layer is the interface between controller and application. The functions developed in this level permit the application level to invoke controller functions. The ADC (Analog Digital Converter) module converts analogical signals from the force sensors to digital, and the SPI (Serial Peripheral Interface) allows communications of the CPU with the accelerometer and the mini-SD card which are not used in this case. In this layer are also included the set of USB and RTC (Real Time Clock) functions, needed for the online monitorization.

- Interface level: this layer contains the main functions that the sensor node performs during its duty cycle. These functions range from reading ADC channels or communicating through the SPI interface, to sending and receiving data from the USB. Programmed interrupt routines functions are also developed in this layer.

- Application level: this is the top level layer and executes related actions according to interruptions received (external switches or internal interruptions).

Once the received data from sensors are processed, a data frame is arranged and prepared for being sending. The structure of this data frame is formed for a header containing an ID with the purpose of identifying the beginning of the frame, followed 
by 4 bytes assigned to the time of measure and a byte which indicates the length of the data information. As Figure 6. shows the data frame enclose information of the measurements taken; the first two bytes give information about the frequency of sampling and the number of sensors measured. According to this last parameter, the rest of bytes corresponding to each sensor in groups of two bytes due to the 10-bit conversion configuration.

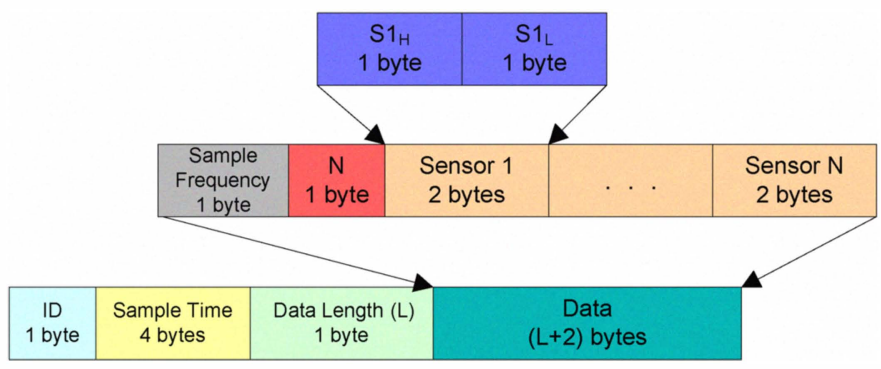

Figure 6. Data Frame

\section{PC APPLICATION}

The PC application has been developed in Visual $\mathrm{C}++$ using the Object Oriented Programming methodology (OOP), which is based in classes. The architecture is show in the Figure 7. There are five blocks, where the most important are the USB process and the graphical routines.

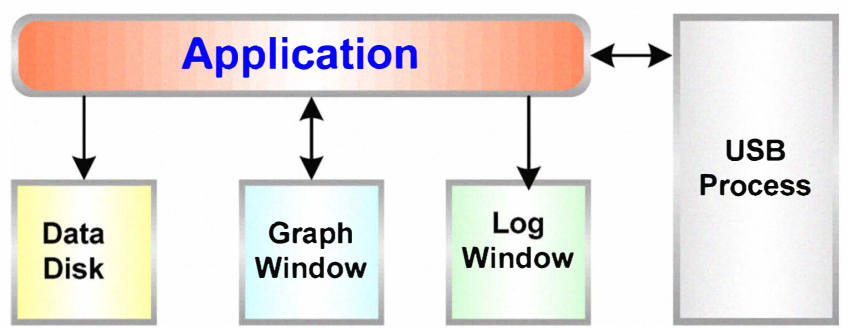

Figure 7. PC Software Architecture

The data obtained by the hardware device after gathering and processing the signals coming from the FSR sensor, are sent via the USB connection to a PC, where an application is running. This application receives the data and visualizes and stores them.

Due the fact that the data rate of the device is low (less than $1 \mathrm{KBps}$ ), the Human Interface Device (HID) protocol has been implemented in the Sensor Device, this way getting an easier method of gathering the data from the PC application, because most operating systems will recognize standard USB HID devices, like keyboards and mice, without needing a special driver

The application has some functionality that makes easier for the doctors the analysis of the data gathered by the hardware device. These functionalities are:
- Start/Stop: this button allows to control the exact moment in which the test starts and ends. When the test stars, a new process is created in the application, which is always controlling the USB communications with the device, and passing the gathered data to the GUI window.

- Zoom: the zooming tool allows visualizing more accurately the signals corresponding to the force applied by the patient's each of the fingers.

- Log: the application allows visualizing a registry or log with the messages corresponding to the events that appear during the testing process (communication states).

- Files: the application allows saving the data in files with ".csv" format, in order to edit and analyze later in a PC program like Excel.

\section{TESTS AND RESULTS}

The obtained results are going to be analyzed separately depending on the test performed in order to obtain them.

\section{A. Hand-grip}

Figure 8. shows a screen capture of the PC application, where a hand-grip force test is being carried out.

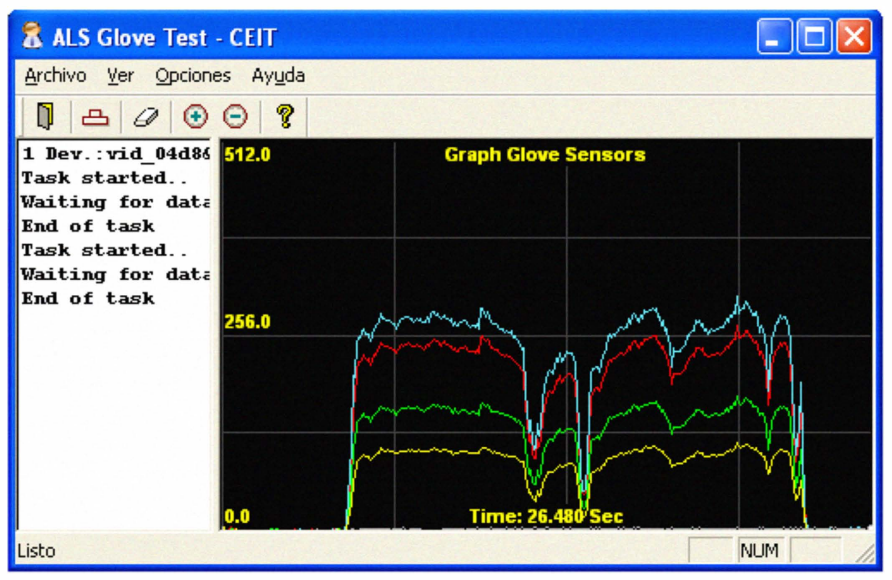

Figure 8. PC application showing hand-grip force test results.

As it can be seen in the figure, the force signal corresponding to each of the fingers is plotted using a different color. That way the analysis of the graph is easier for the clinicians.

\section{B. Finger tapping}

Figure 9. shows a screen capture of the PC application, where a finger tapping test is being carried out.

The main innovation of this system is that it can measure both the frequency of the tapping, but also the force the patient applies when carrying out the test. 
Another key point of this finger tapping test system compared to the existing ones is that the test can be performed using any of the five fingers of the hand. That way, two kind of finger tapping tests can be carried out: one in which the fingers the patient uses most are involved in the testing process, and another one in which the patient uses the fingers that he or she is less keen to use.

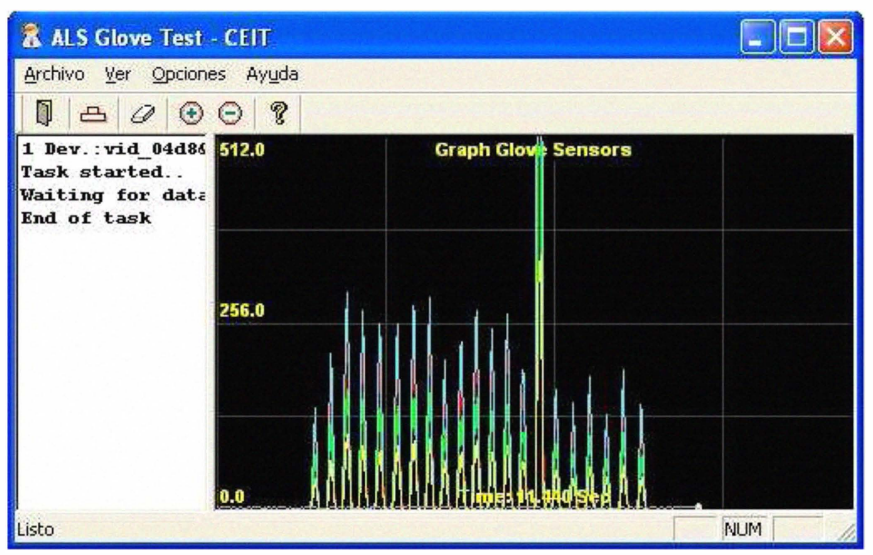

Figure 9. PC application showing finger tapping test results.

\section{CONCLUSIONS AND FUTURE WORK}

The best way to improve the life quality and life expectancy of people affected by neurodegenerative diseases, such as Parkinson Disease and Amyotrophic Lateral Sclerosis, is by improving the treatment of such diseases. And prior to that step, the most important one is to improve the diagnosis and monitoring of the neurodegenerative disease.

Intended to fill the lack of novel systems which provide accurate data about neurodegenerative disease symptoms, a sensor device has been designed and developed. It consists of a multiplication and multipurpose versatile device, which allows carrying out two of the main testing processes described in the literature for the diagnosis and monitorization of PD and ALS: finger tapping test and hand-grip strength test.

For that, a sensorized glove has been designed, and Force Sensitive Resistors (FRS) have used for that purpose. Along with the glove, a tiny and lightweight hardware device has also been developed. The device has been designed in order to comply with requirements of versatility, scalability and flexibility. The same hardware device allows carrying out several types of tests.

Finally a PC application has also been developed, in order to receive and visualize the data gathered by the hardware device. This application acts as interface between the clinician and the system.

The future work which remains to do is focused on the PC application, and basically consists of improving the user interface and offering new capabilities to analyze the data.

One of the main functionality which is planned to be implemented consists of calculating the FFT (Fast Fourier
Transform) of the finger tapping signal. This will offer to the doctors and clinicians a more accurate information about the parameters associated with that signal, and thus, with the test itself. The most valuable parameter to be measured using these technique will be the tapping frequency. This frequency and force of the tapping will be able to be measured with greater accuracy.

The second main task to be carried out is adding the possibility of using cursors when taking measurements from the force vs. time graphs plotted in the application. This will allow the clinicians determining a time period over which the measurement and analysis is going to be done, focusing on the most interesting parts of the whole test

\section{REFERENCES}

[1] K. Kinsella, D. R. Phillips, "Global Aging: The Challenge of Success", Population Bulletin, vol. 60, nr. 1, March 2005

[2] S. von Campenhausen et al., "Prevalence and incidence of Parkinson's disease in Europe", European Neuropsychopharmacology, vol. 15, issue 4, pp. 473-490, August 2005

[3] I. Shimoyama, T. Ninchoji, K. Uemura, "The finger-tapping test: a quantitative analysis", Archives of Neurology, vol. 47, issue 6, pp. 681684, June 1990

[4] D. A. Nowak, "Assessment of Deficient Motor Function of the Hand in Amyotrophic Lateral Sclerosis", Amyotrophic Lateral Sclerosis: New Research, Nova Publishers, pp. 263-277

[5] National Parkinson Foundation - Hoehn and Yahr Staging of Parkinson's Disease, Unified Parkinson's Disease Rating Scale (UPDRS), and Schwab and England Activities of Daily Living; http://www.parkinson.org/Page.aspx?pid=367

[6] M. K. Christianson, J. M. Leathem, "Development and Standardisation of the Computerised Finger Tapping Test: Comparison with other finger tapping instruments", New Zealand Journal of Psychology, July 2004

[7] S. Aitkens et al., "Relationship of manual muscle testing to objective strength measurements", Muscle \& Nerve, vol. 12, issue 3, pp.173-177. Mar. 1989

[8] R. McDermid, "A comparison of alternative devices of the finger tapping test", Archives of Clinical Neuropsychology, vol. 15, issue 8, Page 690, November 2000

[9] Á. Jobbágy a, P. Harcos, R. Karoly and G. Fazekas, "Analysis of fingertapping movement", Journal of Neuroscience Methods, vol. 141 (2005), pp. 29-39

[10] http://www.reitanlabs.com/catalog/default.php

[11] M. Soichir et al., "Quantitative analysis of cerebellar ataxia with a finger tapping device containing a pressure sensor", Neurological Medicine, vol.61, issue 1; pp. 99-101 (2004)

[12] M. Yokoe et al., "Opening velocity, a novel parameter, for finger tapping test in patients with Parkinson's disease", Parkinsonism Related Disorders, vol. 15, issue 6, pp. 440-4

[13] R. Okuno et al., "Measurement system of finger-tapping contact force for quantitative diagnosis of Parkinson's disease", 29th Annual International Conference of the IEEE Engineering in Medicine and Biology Society, EMBS 2007, pp. 1354-1357, 22-26 Aug. 2007

[14] Vernier Software \& Technology http://www.vernier.com/probes/hdbta.html

[15] C. Long, "Intrinsic-extrinsic muscle control of hand in power grip and precision handling - an electromyographic study", Journal of bone and joint surgery - American volume, vol. 52, issue 5, pp. 853-\&

[16] Tekscan, Inc. http://www.tekscan.com/flexiforce/flexiforce.html 